\title{
Research for Image Haze-Removal Algorithm Using the Dark-Channel Prior Based on Wavelet Transform
}

\author{
Wu Sijiu ${ }^{1, *}$, Zhang Haiyan ${ }^{2}$ and Cheng Weidong ${ }^{1}$ \\ ${ }^{1}$ Chengdu University of Information Technology, Chengdu, 610225, PR China; ${ }^{2}$ Chengdu Aeronautic Polytechnic and \\ Technical College, Chengdu, 611730, PR China
}

\begin{abstract}
In this paper, having compared the image haze-removal algorithms for image restoration and image enhancement, the principle of image haze-removal algorithm using dark channel prior is analyzed and discussed. The shortcomings of the algorithm are pointed out. At the same time, image haze-removal algorithm using the dark-channel prior based on wavelet decomposition is proposed. There are three steps included: three channel wavelet transform for the image, image haze-removal algorithm using the dark-channel prior of the low frequency components for dark channel prior and upgrading sharpening for high frequency components. The experimental results show that the algorithm can improve the resolution of image after image haze-removal and have better performance in detail, but also can effectively reduce the running time, improve the operating speed.
\end{abstract}

Keywords: Dark channel prior, image haze-removal, image, wavelet.

\section{INTRODUCTION}

Images taken daily are influenced by atmospheric fog, dust and other suspended particles. It is very difficult to computer for its visual application such as surveillance, tracking and navigation system. Besides these suspended particles of atmosphere can reduce the visibility, light attenuation, and affect the scattering, reflection caused by the particles itself, it results in a decline in attenuation characteristic of color as for the acquisition to image contrast, brightness and resolution. Therefore, it has important theoretical significance and practical application value to the atomization image by image haze-removal and enhancing method, and to eliminate the effect of suspended particles in the atmosphere for the target image.

In earlier time, while comparing various images hazeremoval, some basic parameters [1] are obtained such as the depth of field, atmospheric visibility et al, and then restore the atomization image according to these parameters. But it is not applicable for single image haze-removal. Until now, researchers have studied on single image haze-removal. To sum up, there are two kinds of methods proposed: one is based on the image enhancement. The literature [2] shows homomorphic filtering for image haze-removal. It will be as the high-frequency part for restoring the area, while the other will be as the low-frequency part for haze-removal. After discriminating the high-frequency from the low-frequency part, image haze-removal can achieved by reducing the lowfrequency and/or enhancing the high-frequency. Literature [3] proposed the histogram equalization for processing atomization image. It can improve the image haze-removal obtained under some mist weather conditions. In literature [4], based on improvement of histogram equalization and with normal distribution solution for sky area, the histogram equalization for the local area is carried by using the mobile template.

The other is based on the image restoration. The method focuses on the principles of the formation as reduced-quality image taken in the mist weather, and on combining with the physical model to atmospheric physical scattering to carry image haze-removal. The main is image haze-removal algorithm using the dark-channel, and so on. Having analyzed a large number of images without haze, Literature [5] has found out that there is a dark channel area for all the images and the pixel value of dark channel area is zero. As a pixel of image covered by mist is filled with that of mist, it can be restored the original image by analyzing the area and estimating ambient light and atmospheric light.

There are quite different image haze-removal thoughts between image enhancement and image restoration. Image enhancement is essentially to enhance the image contrast and to correct the color. While it does not study effective formation mechanism and provide effective compensation for image taken in mist, the effect of image haze-removal is limited. Image restoration is that the image restoration model, which is established on the law of atmospheric physical scattering, needs to obtain parameters to restoration such as atmospheric light and environmental light, scene depth, the refractive index of air and so on [6-8].

Comparing with experimental images processed by the methods mentioned above, the image restoration processed by the histogram equalization method is good and its details are closer to reality under haze weather condition. While in the fog condition, the histogram equalization is not applicable because most of image processed displays gray. In the 


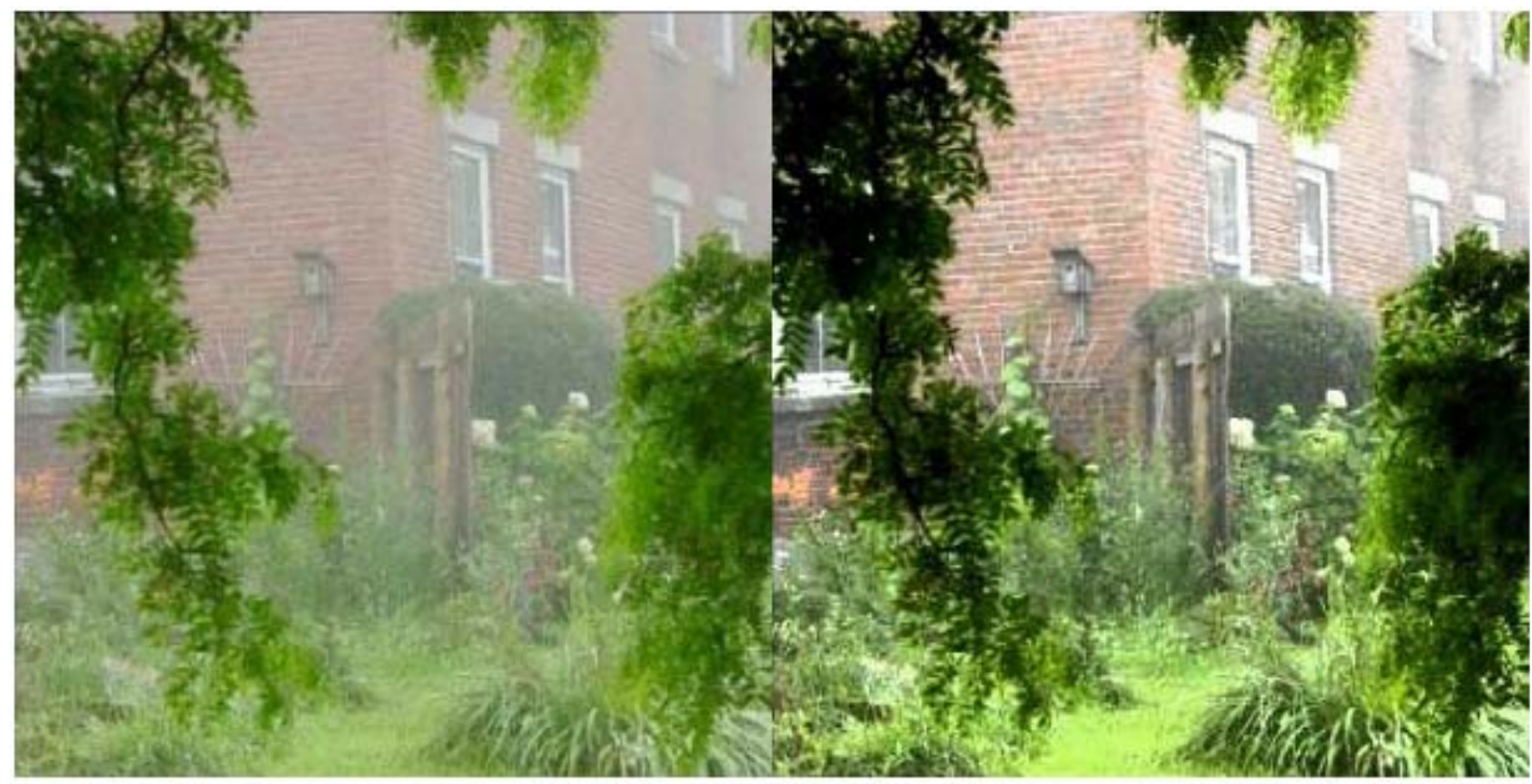

(a) Original image

(b) Image processed by histogram equalization

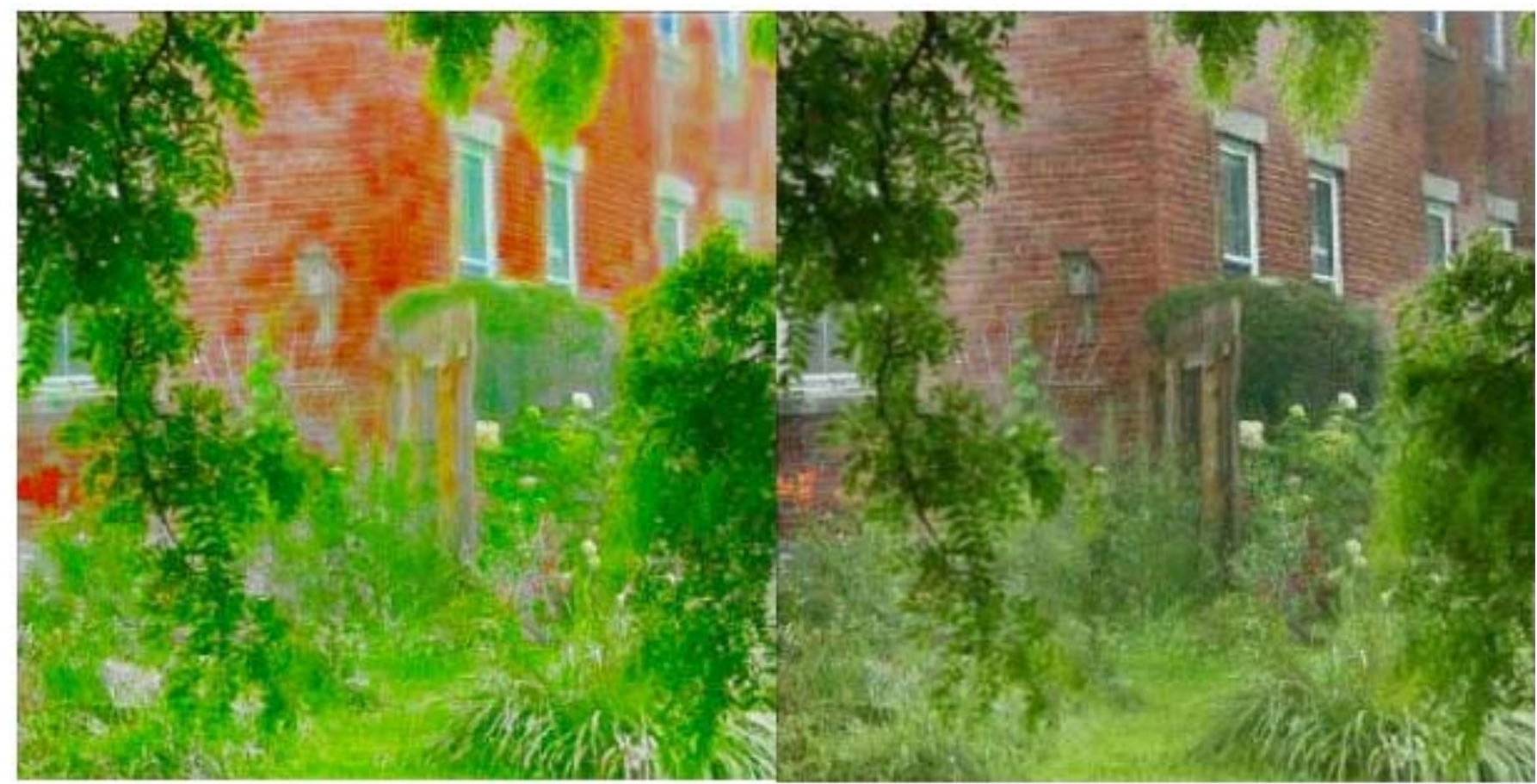

(c) Image haze-removal by homomorphic filtering

(d) Image haze-removal by using the dark-channel prior

Fig. (1). Comparison of the effect of image haze-removal.

case, the gray of image is to stretch to the entire image, and the saturation distortion of image occurs obviously by using histogram equalization. The image restoration processed by homomorphic filtering method is also good under mist. These parts covered by fog of the image taken change slowly, as can be regarded as the low frequency part of the image. The image haze-removal can be reached by reducing lowfrequency components and/or by enhancing high-frequency component. But the effect for image haze-removal is very poor under fog conditions. The image, obtained by homomorphic filtering method, is too saturated that there is image distortion in some areas of the image.

The image color obtained by image haze-removal algorithm using the dark-channel prior is more real, its details are true as well as the whole is satisfied. With this method, the operation speed is too slow because of calculating on the transmittance and optimization. It is relatively time-consu- 
ming method by which is required to compare a pixel to the other in order to calculate the dark-channel area of image. In the specific course of the experiment, in order to obtain the dark-channel area of image, it is a large amount of calculation for comparing every pixel to the other. This paper will introduce image haze-removal algorithm using the darkchannel prior and wavelet transform briefly. By combining with the advantages of wavelet transform and image hazeremoval using dark channel prior method, it is to realize fast image haze-removal.

\section{IMAGE HAZE-REMOVAL USING DARK CHAN- NEL PRIOR METHOD}

\subsection{Atmospheric Scattering Physical Model}

According to the atmospheric scattering physical model presented in literature [6], fog image can be expressed as:

$I(x)=J(x) t(x)+A(1-t(x))$

$I$ represents the foggy image taken usually, $J$ the image to get with no fog, $A$ the ambient light component, and $t$ the transmissivity. Image haze-removal is to get image with no fog $J$ from $I$. The first item $J(x) t(x)$ in the equation is for direct attenuation, the second $A(1-t(x))$ is for the atmospheric optical components.

\subsection{Dark Channel Prior Method}

Having analyzed a large number of images without haze, Literature [5] has found out that the most of these images has areas for at least one color channel of three chan$\operatorname{nels}(\mathrm{R}, \mathrm{G}, \mathrm{B})$, which has very low pixel value. That is to say, the area light intensity value is a very low. Here, image $\mathrm{J}$ is defined as:

$J^{d a r k}(x)=\min _{c \in\{r, g, b\}}\left(\min _{y \in \Omega(x)}\left(J^{c}(y)\right)\right)$

Here $J^{c}$ is the color channel of $\mathrm{J}, \Omega(x)$ is the dark channel area. Study shows that intensity of dark channel image area close to 0 . $\mathrm{J}$ represents no fog image, and $J^{\text {dark }}$, the dark channel of $\mathbf{J}$ of no fog image in Equation (2). The rule that low pixel area exists in the image color channel mentioned above is referred collectively to as the dark channel prior.

\subsection{Image Haze-Removal Algorithm Using the Dark- Channel}

Any images are subject to the impact of the surrounding environment. Their atmospheric optical A is always greater than 0 , and the transmissivity at the same area of image is basically the same. Suppose a minimum value given to both sides of Equation (1) at the same time, the following is available:

$\min \left(y \in \Omega(x) \frac{I^{c}(y)}{A^{c}}\right)=\tilde{t}(x) \min _{y \in \Omega(x)}\left(\frac{J^{c}(y)}{A^{c}}\right)+(1-\tilde{t}(x))$

After the minimum operation taken for three channels of R, G, B, we get:

$\min \left({ }_{c} \min _{y \in \Omega(x)}\left(\frac{I^{c}(y)}{A^{c}}\right)\right)=\tilde{t}(x) \min _{c}\left(\min _{y \in \Omega(x)}\left(\frac{J^{c}(y)}{A^{c}}\right)\right)+$ $(1-\tilde{t}(x))$

Because dark channel of no fog image tends to 0, i.e.:
$J^{\operatorname{dark}}(x)=\min _{c}\left(\min _{y \in \Omega(x)}\left(J^{c}(y)\right)\right)=0$

And $A^{c}$ is always positive, so that

$\min _{c}\left(\min _{y \in \Omega(x)}\left(J^{c}(y)\right)=0\right.$

Substitute Equation(6) into Equation (4), transmissivity t can be obtained:

$\tilde{t}(x)=1-\min _{c}\left(\min _{y \in \Omega(x)}\left(\frac{I^{c}(y)}{A^{c}}\right)\right)$

The image looks not very authentic as image hazeremoval is totally realized in the image haze-removal process, and loses the sense of depth. In order to retain part effect of the distance mist, a constant is introduced in the Equation above:

$\tilde{t}(x)=1-\omega \min _{c}\left(\min _{y \in \Omega(x)}\left(\frac{I^{c}(y)}{A^{c}}\right)\right)$

We can get the basic estimation method of refractive index by the Equation above. It is not very precise. In Literature [5], distribution function of image transmissivity $t(x)$ is given by application of soft matting. Transmissivity $t$ can be acquired through solutions of the following equation

$(L+\lambda U) t=\lambda \tilde{t}$

$\lambda$ is the parameter introduced, $\mathrm{L}$ is the Laplasse tracing matrix. After calculating transmissivity $t(x)$, and as long as estimating the atmospheric optical value $\mathrm{A}, J(X)$ can be recovered by substituting the transmissivity and value $\mathrm{A}$ into Equation (1):

$J(x)=\frac{I(x)-A}{\max \left(t(x), t_{0}\right)}+A$

Estimation method of atmospheric optical A is as the following: In the original image, the maximum value of brightness located the top $0.1 \%$ is as the value of $A$ according to the brightness of the pixel points descending.

\subsection{Image Wavelet Transform}

To the fog image, the area affected by fog is with high brightness generally and its tone changed slowly. Compared with the other areas, there are high energy and less variance. As considering for frequency domain, because there is smooth tone of the fog image and relative indistinct compared its details to with that of no fog image, the energy of fog affects on the low frequency part the image while the detail of the scene is mainly distributed in the high frequency part of the image. Therefore, image haze-removal algorithm using the dark-channel prior can be used for removing the effects of fog for the low frequency part. At the same time, it is sharpened with moderate enhancement for the high frequency part to promote detail information of the scene. The image haze-removal can be reconstructed after treatment of the high-frequency and low-frequency part. The Mallat algorithm is used to the wavelet transform, and its principle is expressed as follows:

Here, three "two-dimensional wavelet" is defined in Equation (11), in which $v_{j}^{2}(j \in Z)$ is separable multire solution analysis of $L^{2}\left(R^{2}\right)$. We suppose: 
$\emptyset(\mathrm{x}, \mathrm{y})=\varnothing(\mathrm{x}) \emptyset(\mathrm{y})$ is the corresponding two dimensional scaling function, while $\varphi(x)$ is a one-dimensional standard orthogonal wavelet which corresponds scale function.

$\left\{\begin{array}{l}\varphi^{1}(x, y)=\emptyset(x) \varphi(y) \\ \varphi^{2}(x, y)=\varphi(x) \emptyset(y) \\ \varphi^{3}(x, y)=\varphi(x) \varphi(y)\end{array}\right.$

We can get:

$\left\{\begin{array}{l}2^{-j} \varphi^{1}\left(2^{-j} x-m, 2^{-j} y-n\right) \\ 2^{-j} \varphi^{2}\left(2^{-j} x-m, 2^{-j} y-n\right) \\ 2^{-j} \varphi^{3}\left(2^{-j} x-m, 2^{-j} y-n\right)\end{array}\right.$

Is respectively the standard orthogonal basis within $L^{2}\left(R^{2}\right)$. Suppose $f=f(x, y) \in v_{j}^{2}$ is as image signal to be analyzed. The two-dimensional approximation image is:

$A_{j} f=A_{j+1} f+D_{j+1}^{1} f+D_{j+1}^{2} f+D_{j+1}^{3} f$

In which

$A_{j+1} f=\sum_{m=-\infty}^{\infty} \sum_{n=-\infty}^{\infty} C_{j+1}(m, n) \emptyset_{j+1}(m, n)$

$D_{j+1}^{i} f=\sum_{m=-\infty}^{\infty} \sum_{n=-\infty}^{\infty} D_{j+1}^{i}(m, n) \emptyset_{j+1}(m, n), i=1,2,3$

According to the orthogonality of the wavelet function and scale transform, we also get by Equation (11), Equation (12) and Equation (13):

$C_{j+1}(m, n)=$

$\sum_{k=-\infty}^{\infty} \sum_{l=-\infty}^{\infty} h(k-2 m) h(1-2 n) C_{j}(k, l)$

$\left\{\begin{array}{l}D_{j+1}^{1}(m, n)=\sum_{k=-\infty}^{\infty} \sum_{l=-\infty}^{\infty} h(k-2 m) g(1-2 n) C_{j}(k, l) \\ D_{j+1}^{2}(m, n)=\sum_{k=-\infty}^{\infty} \sum_{l=-\infty}^{\infty} g(k-2 m) h(1-2 n) C_{j}(k, l) \\ D_{j+1}^{3}(m, n)=\sum_{k=-\infty}^{\infty} \sum_{l=-\infty}^{\infty} g(k-2 m) g(1-2 n) C_{j}(k, l)\end{array}\right.$

Here the matrix operator is introduced. $H_{r}$ and $H_{c}$ representsrespectively the rows effect operator and the columns effect operator of arrays $\left\{C_{k, l}\right\}_{(k, l) \in Z^{2}}$ from scale filter coefficients, while $G_{r}$ and $G_{c}$ representsrespectively the rows effect operator and the columns effect operator of arrays $\left\{C_{k, l}\right\}_{(k, l) \in Z^{2}}$ from wavelet filter coefficients. The twodimensional Mallat decomposition algorithm is as follows:

$$
\left\{\begin{array}{l}
C_{j+1}=H_{r} H_{C} C_{j} \\
D_{j+1}^{1}=H_{r} G_{C} C_{j} \\
D_{j+1}^{2}=G_{r} H_{C} C_{j} \\
D_{j+1}^{3}=G_{r} G_{C} C_{j}
\end{array}\right.
$$

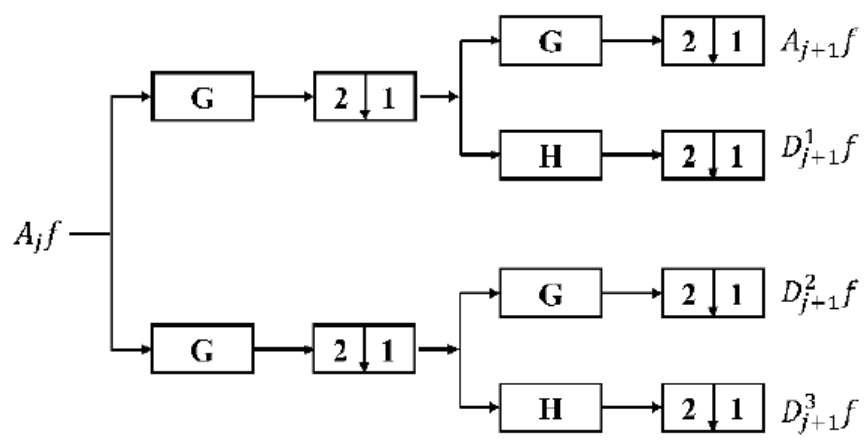

Fig. (2). Structure diagram of two-dimensional Mallat wavelet transform algorithm.

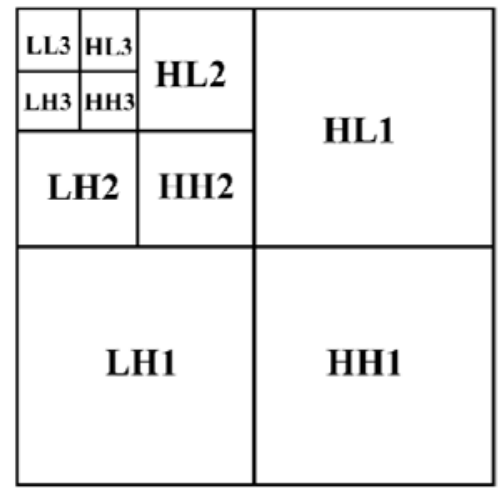

Fig. (3). Schematic diagram of three-stage wavelet decomposition of image.

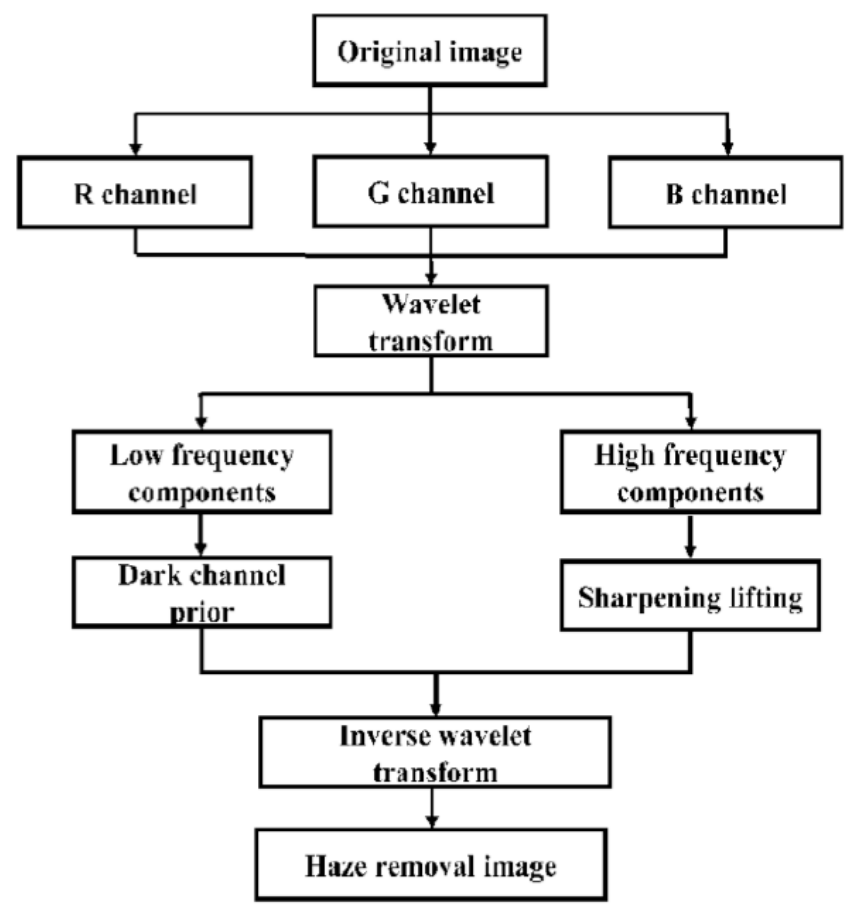

Fig. (4). Flow chart of image haze-removal.

The structure diagram of two-dimensional Mallat wavelet transform algorithm is shown in Fig. (2). It can be seen from the figure that the wavelet transform decompose the image signals into a plurality of low frequency sub-band component. And each layer wavelet transform decompose the upper layer image into 4 sub-bands, which have a low frequency 


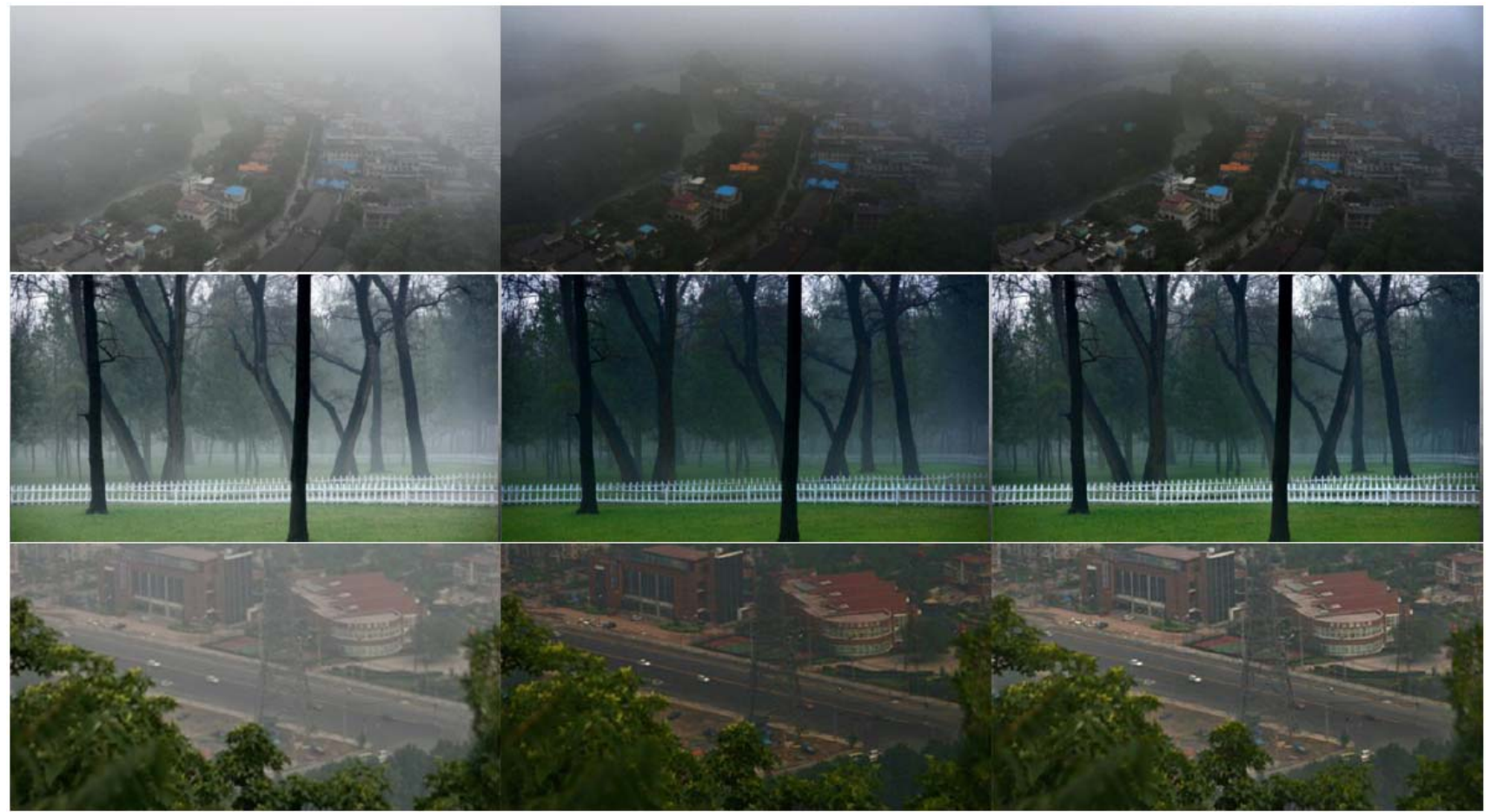

(a) Original image

(b) Image haze-removal using dark-channel prior (c) Image haze-removal mentioned in this paper

Fig. (5). Comparison of two methods for image haze-removal.

band LL and three high frequency bands with the direction selectivity such as HL, LH, and HH. The next layer wavelet transform proceed with decomposition on the band LL. The multistage decompositions are obtained. A schematic diagram of three-stage wavelet decomposition of image is shown in Fig. (3). By the Pyramid structure, it can be isolated well the representation of low frequency information for image content from that of high frequency information for image details. Therefore, it is for image haze-removal algorithm using the dark-channel prior with the low frequency part and for filtering of sharpening with the high frequency part. The algorithm flow as shown in Fig. (4).

\subsection{Promote Sharpening with the High Frequency}

The gain function for nonlinear promotion is used in this paper. In order to achieve promoting sharpening, the modification for the high-frequency wavelet coefficients is proposed by threshold functions. The threshold functions are as follows:

$C_{t h r}(I)=\left\{\begin{array}{c}-a \times t h r^{2}+t h r+C(I) C(I)<-t h r \\ a \times C(I)^{2}|C(I)|^{2} \leq t h r \\ a \times t h r^{2}-t h r+C(I) C(I)>t h r\end{array}\right.$

Here, $C_{t h r}(I)$ is for the high frequency coefficient modified by threshold, $C(I)$ is as the original high frequency coefficient and $a=\sqrt[T]{t h r}$ as the gain factor. If fog is thick fog, $T=2$, while the fog is less thin, $T$ is properly increasedto reduce the image distortion.

The standard deviation of low frequency sub-band increases with the number of decomposition levels. It shows that the most energy of the image gathered in the low frequency band. The less energy is contained with the increasing frequency. It also shows that there is better wavelet coefficient energy concentration and wavelet coefficients of the lower level have a more important position.

\section{EXPERIMENTAL RESULTS AND ANALYSIS}

In order to verify the effectiveness of the algorithm, 6 groups of fog image processing are chosen which are for different scenery, different illumination and different contrast. Respectively, comparing the dark channel prior with the algorithm mentioned in this paper, the experimental results are as shown in Figs. (5 and $\mathbf{6})$.

As can be seen from Fig. (5), fog effect can be eliminated effectively by the algorithm mentioned in this paper and/or the dark channel prior. The fog image after haze-removal by the dark channel prior algorithm is darker with lower brightness. It is especially obvious in the third set of results in Fig. (5). There is slightly difference of the algorithm mentioned in this paper for color correction from the haze-removal by the dark channel prior algorithm. The second sets of results in Fig. (5), the recovery of the fence white is impure by dark channel prior algorithm, and with slightly blue, while results by the algorithm mentioned in this paper is relatively good.

In order to observe, after local amplifying the results of three image groups in Fig. (6), it can be seen that maintaining of the details by the algorithm mentioned in this paper is better than that by dark channel prior. The image of cars and buildings is more clear and bright. And texture details of vegetation obviously is not vague. 

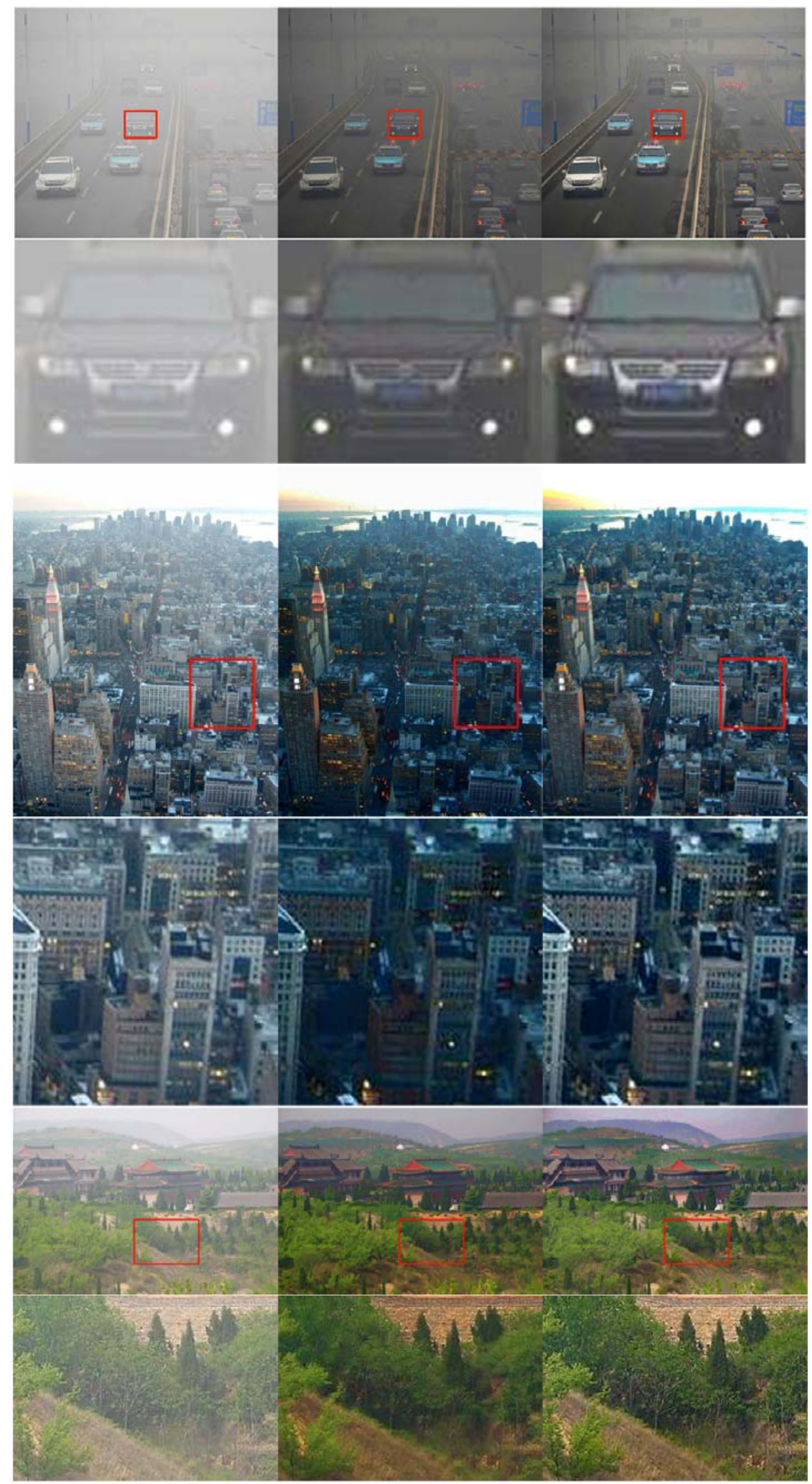

(a) Original image

(b) Image haze-removal using

(c) Image haze-removal mentioned

dark-channel prio

$$
\text { in this paper }
$$

Fig. (6). Local image amplification to comparison of two methods for image haze-removal (The local image amplification in even numbered columns is for the image of red box in the corresponding forefront columns). 
Table 1. Processing time comparisons of algorithms (unit: s).

\begin{tabular}{|c|c|c|c|c|c|c|}
\hline Image & $\mathbf{5 ( a )}$ & $\mathbf{5 ( b )}$ & $\mathbf{5}(\mathbf{c})$ & $\mathbf{6 ( a )}$ & $\mathbf{6 ( b )}$ & $\mathbf{6}(\mathbf{c})$ \\
\hline \hline $\begin{array}{c}\text { Image haze-removal using } \\
\text { dark-channel prior }\end{array}$ & 33.76 & 41.33 & 15.16 & 27.59 & 27.46 & 43.92 \\
\hline Method mentioned in this paper & 5.61 & 6.96 & 2.87 & 4.65 & 4.60 & 7.43 \\
\hline
\end{tabular}

In order to compare timeliness of algorithms, the simulation for 6 image groups in Matlab R2013a is carried in our experiments. The hardware platform is composed of CPU core 2 Duo 3.30GHZ, memory 8GB RAM. The use of the time is as shown in Table $\mathbf{1}$. The image sequence is according to Figs. (5 and $\mathbf{6}$ ). Compared to the standard image hazeremoval using dark channel prior algorithm, it can be seen that the computational complexity of the algorithm mentioned in this paper is greatly reduced. And the validity of the algorithm is improved.

\section{CONCLUSION}

The mist effect on images reduces mainly the degradation of color information and the image contrast. As considering these two aspects, the image haze-removal using darkchannel prior is used for the restoration of color information; while the high-frequency sharpening filter of wavelet transform is use for enhance image contrast by the algorithm mentioned in this paper. Also better visual effect can be obtained by the algorithm from experiments. The effect of haze-removal is more obvious, the details of the image are color richer and the color more real. Because the color information in the wavelet low frequency component is recovered, so this algorithm in computing speed is greatly improved.

The main advantage of this algorithm is a simple method with fast speed. It may achieve good effect. There are some shortcomings of algorithm itself, such as the lower accuracy of estimating ambient light, effect of restoring image under heavy fog condition. The levels given to wavelet transform will affect directly image haze-removal. The further algorithm may be supplied to find a more effective, suitable model and parameters for better effect.

\section{CONFLICT OF INTEREST}

The authors confirm that this article content has no conflict of interest.

\section{ACKNOWLEDGEMENTS}

Declared none.

\section{REFERENCES}

[1] J. P. Oakly, and B. L. Satherley, "Improving image quality in poorvisibility condition using a physical model for contrastdegradation," IEEE Transactions on Image Processing, vol. 2, no. 7, pp. 167-179, 1998.

[2] G. Chen, T. Wang, and H. Zhou, "A novel physics-based method for restoration of foggy day images", Journal of Image and graphics, vol. 13, no. 5, pp. 888-893, 2008.

[3] O.Y. Kim, 1. S. Kim, and S.H. Hwang, "An advanced contrast enhancement using partially over lapped Sub-Block histogram equalization," In: IEEE Transactions on Circuits and Systems for Video Technology, 2001, pp. 475-484.

[4] P. Zhou, H. Zhu, and X. Qian, "An image clear mess method for fog," Normal of Image and Graphics, vol. 1, pp. 126-130, 2004.

[5] K. M. He, J. Sun, and X. Tang, "Single image hazeremoval using dark charnel prior," In: Proceedings of IEEE Conference on Computer Vision and Pattern Recognition (CVPR), Miami, FL, USA: IEEE Computer Society, 2009, pp. 1956-1963.

[6] E. J. Mc Cartney, Optics of Atmosphere: Scattering by Moleculesand Particles, New York: John Wiley and Sons, 1996, pp. 2332.

[7] S. Mallat, "A theory for multiresolution signal decomposition: The wavelet representation," IEEE Transactions on PAMI, vol. 7, no. 11, pp. 674-693, 1989.

[8] S. G. Narasimhan, and S. K. Chromatic, "Framework for vision in bad weather," In: Proceedings of the IEEE Conference on Computer Vision and Pattern Recognition, 2000. 